\title{
STATIC AND DYNAMIC BEHAVIOUR OF MODULAR TOOLING SYSTEM FOR VERTICAL-SPINDLE GRINDING MACHINE
}

\author{
BY \\ S. HUZEM \\ Department of Production Engineering, Faculty of Engineering, \\ Mansoura University, Mansoura, EGYPT.
}

\section{السلوك الأستاتيكى والايناميكى لمنظومة عدة معيارية لماكية تجليخ

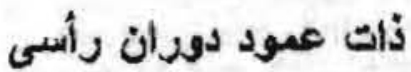

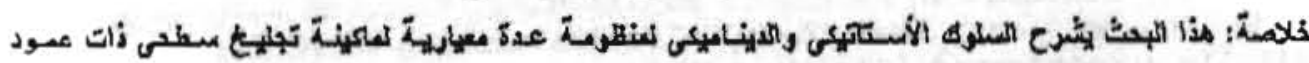

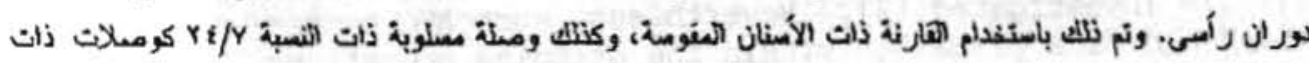

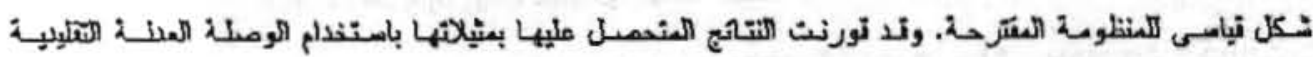

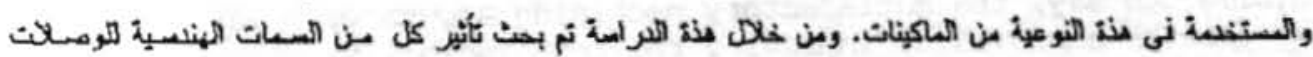

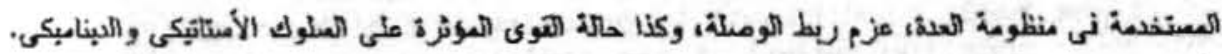

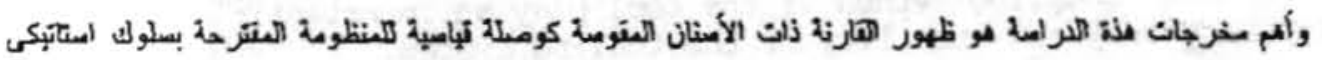

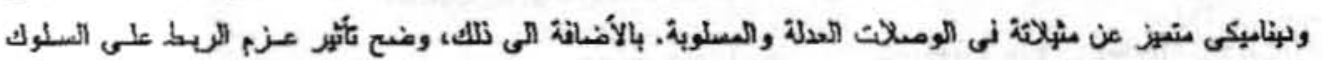

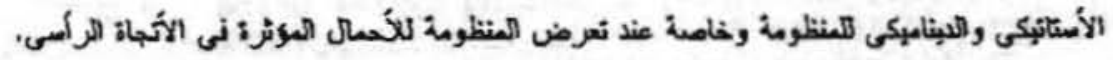

ABSTRACT: This paper describes the static and dynamic behaviour of a proposed tooling system, for vertical-spindle surface grinding machine using, as joint modules, the curvic coupling as vell as a taper joint of the ratio $7 / 24$. The experimental results obtained vere compared with that of the conventional mounting method of flange type (flat joint). Through this work, the effects of the joint geometrical features, joint tightening torque, and loading condition on the static and dynamic behaviour vere investigated.

The most important finding is that the curvic coupling as a joint module for the proposed tooling system, has better static and dynamic behaviour than the corresponding taper and flat joints. Improvement rates of about 308-60\% in the static bending stiffness, about 178-50\% in the damping capacity, and about 98 in the natural frequency, have been observed. In addition, the tightening torque considerably affects the static and dynamic behaviour of the tooling system, particularly when the system is subjected to the vertical loading condition.

\section{INTRODUCTION:}

Recently, owing to the orientation toward individuality and pursuit of convenience and comfort in the market enviroment, product demand has become increasingly diversified, accordingly, product varieties have increased and 
product life cycles have progressively become shorter. Moreover, the rapid pace of technological innovation is being manifested in demand for higher product quality and lower prices.

In this regard, among the various types of machining processes, grinding operation demands particular high-precision, high-efficiency and high-quality. Therefore, in addition to the proper selection of the machining conditions, the performance of the machine and its tooling system is extremely important. With respect to the automatic tool changing (ATC) systems, numerous tooling systems have been tried and developed [for example 1-51, of them the $7 / 24$ taper connection is the most popular tool changing system. On the other hand, the toothed coupling, i.e. the curvic coupling, has claimed an excellent positioning repeatability and high resistance to torsional load $[6-8]$, also its meshing mechanism has been theoretically investigated [9]. Neverthless, such ATC systems have not yet found the field of application within the grinding operations.

Nedless to say, the satisfactory machining accuracy, using such ATC systems, can be achieved by improving the reproducibility of the locating accuracy of the tool, simultaneously ensuring the high joint stiffness. In addition, the coupling method of the tool play an important role to the machining accuracy and stability of the tooling system.

In this paper, therefore, a tooling system using the curvic coupling, has been proposed in the form of the vertical-spindle of a surface grinding machine. In due course, the static and dynamic behaviour of the proposed tooling system have been experimentally investigated. The experiments were carried out also on the conventional tooling system (flange type) and an accommodated $7 / 24$ taper system. Through this work, the effects of the coupling types and coupling tightening torque on the static and dymamic behaviour, under the horizontal and vertical loading conditions, have been clarified.

\section{EXPERIMENTAL PROCBDURE:}

To clarify the static and dynamic behaviour of the proposed tooling system, the vertical-spindle of a surface grinder has been such modified as shown in Fig.1, to accommodate the different coupling modules. In Fig.1, the modified internal thread $M 28 \times 2.0$ of the spindle nose has been used to locate an adapter on which one member of the curvic coupling has been bolted by means of six M5 tightening bolts, while the other member of the curvic coupling has been bolted to the flange. The draw bolt, as it was screwed to the flange through the thread M16, enables the curvic coupling members to be meshed. Then the required tightening torque was applied by means of a torque wrench to the required value. The curvic coupling jointing method can be replaced with either the taper joint or the equivalent flat joint, as shown in Fig.1-b, depending on the experimental procedure.

In the static experiments, the bending stiffness was investigated in 
both the horizontal and vertical directions, with the bending load of up to $500 \mathrm{~N}$, which was applied at the flange through the load cell which was fixed to the table of the grinding machine. The resultant deflections, at the point opposite to the loading point, were detected by means of a dial gauge with 2 um sensitivity, and the measured values were processed to obtain the required static bending stiffness.

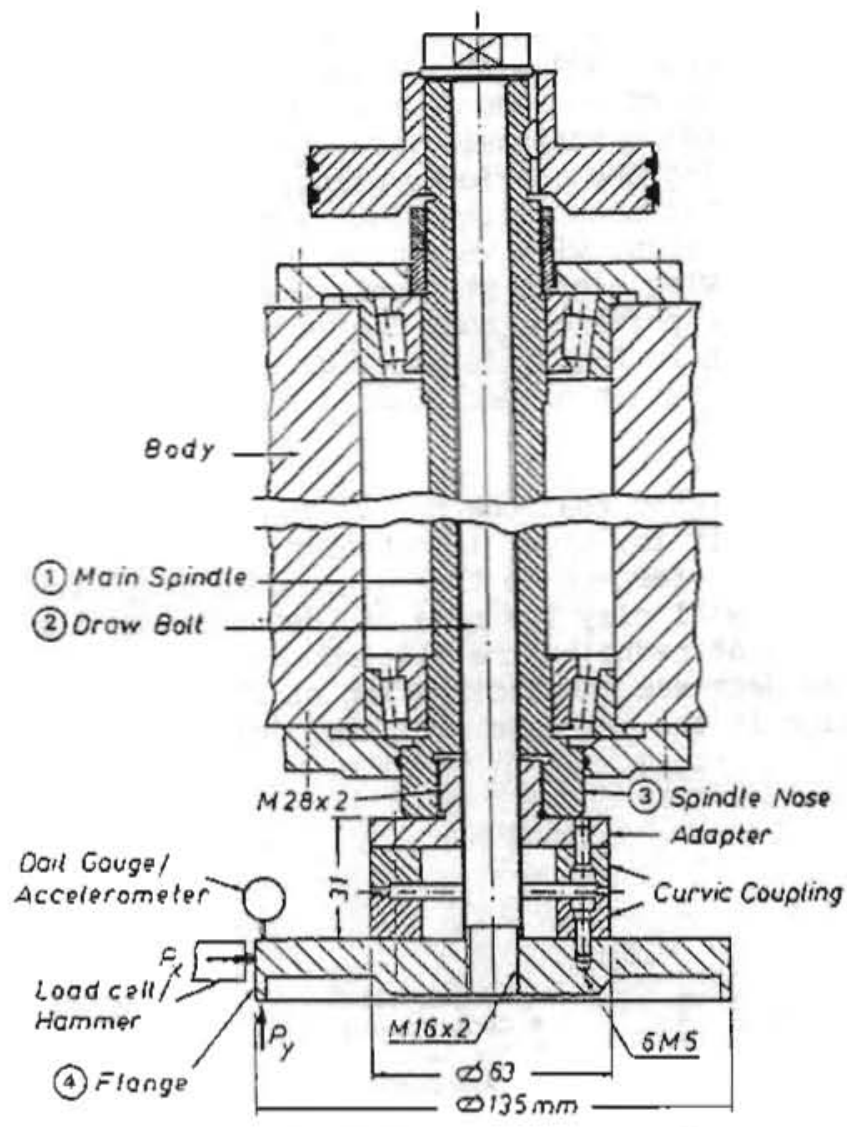

(a) Main spindle drive with curvic coupling.

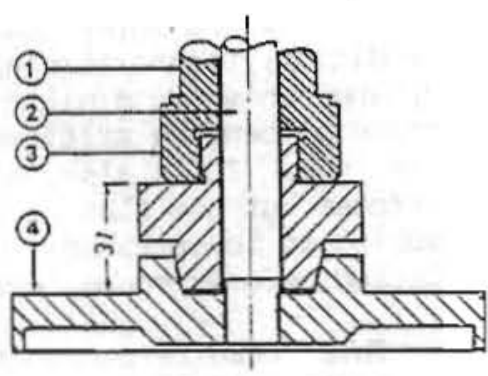

$7 / 26$ Taper Joint

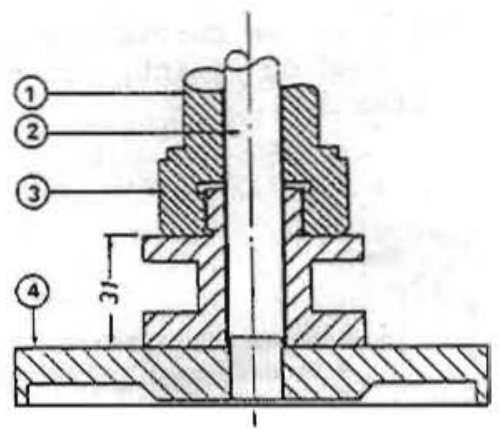

Flat joint

(b) Main spindle drive with other joint modules.

Fig. 1 Schematic view of the experimental set-up.

In the dynamic experiments, the load cell and the dail gauge were replaced with the impulse hammer and peizoelectric accelerometer. The dynamic characteristics were analyzed using the spectrum analyzer, and the damping capacity, measured as the damping ratio, was calculated by the band-width method [10]. Through all the static and dynamic experiments, the tightening torque of the draw bolt has been varied from $25 \mathrm{~N} . \mathrm{m}$ to 200 N.m, by $25 \mathrm{~N} . \mathrm{m}$ step. 


\section{STATIC BEHAVIOUR OF THE TOOLING SYSTEM:}

In surface grinding operation, when the tooling system is subjected to the actual cutting forces, the horizontal and vertical cutting forces are the main components to be considered. In Figs.2 and 3, thus, the effect of the tightening torque on the horizontal and vertical bending stiffness are shown respectively. It is noticeable that for all coupling modules, the bending stiffness increases with the tightening torque. In this regard, within the used range of the tightening torque, the horizontal bending stiffness increased with the rate of about $40 \%$ with the taper joint, while it was about $50 \%$ for both the flat joint and the curvic coupling. The rates of increase in the vertical bending stiffness were about $70 \%, 55 \%$, and $145 \%$ for the taper joint, flat joint and curvic coupling, respectively. In addition, the horizontal bending stiffness of the flat joint and curvic coupling showing similar characteristics, with very close values of the horizontal bending stiffness, and showing higher stiffness than that of the taper one. It is also interesting to point out that the vertical bending stiffness of the flat joint, as shown in Fig.3, is superior to the curvic coupling at lower tightening torques, but at higher tightening torques this relation becomes to be inverse.

This results can be such interpreted that the curvic coupling is one of the typical machine tool joints. It is, thus, important to prevent any separation of the joint surfaces. In respect to the curvic coupling, the tilting motion of the lower member will play the role of determining the joint stiffness. This means it that reducing the tilting of the lower member of the curvic coupling can decrease the inclination of the flange at the loading point, it resulting in the increase of the joint bending stiffness.

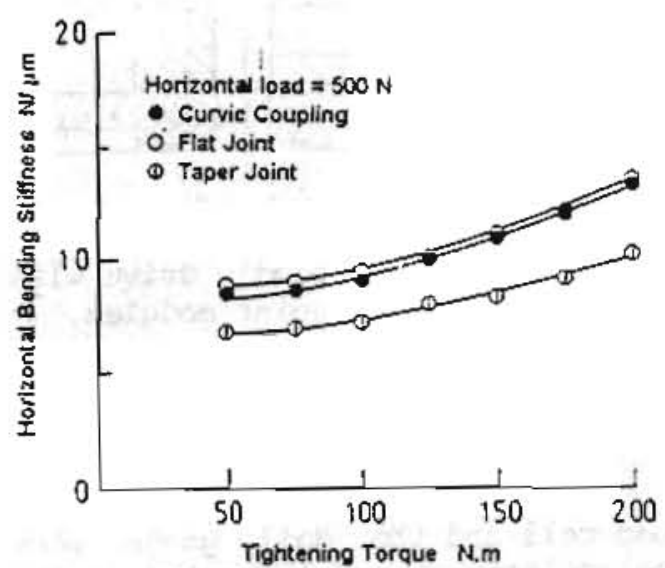

Fig.2 Effect of the tightening torque on the horizontal bending stiffness.

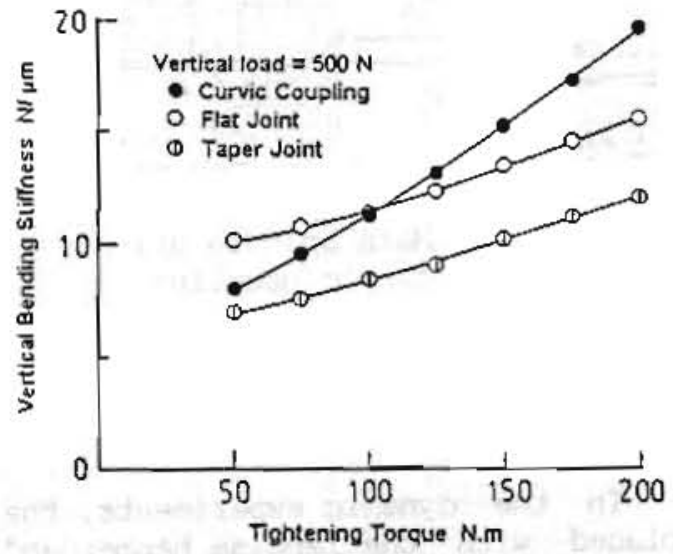

Fig. 3 Effect of the tightening torque on the vertical bending stiffness. 
DYNAMIC BEHAVIOUR OF THE TOOSING SYSTEM:

To clarify the dynamic behaviour of the proposed tooling system, the impulsive excitation technique has been carried out using the same experimental test-rig shown in Fig.1.

Fig. 4 shows a typical example of the frequency response, in the horizontal and vertical directions, of the proposed tooling system with curvic coupling. From which the natural frequency of the major peak, as well as the damping ratio using the band-width method, have been used to clarify the dymamic behaviour of the different joint modules employed in the proposed tooling system.
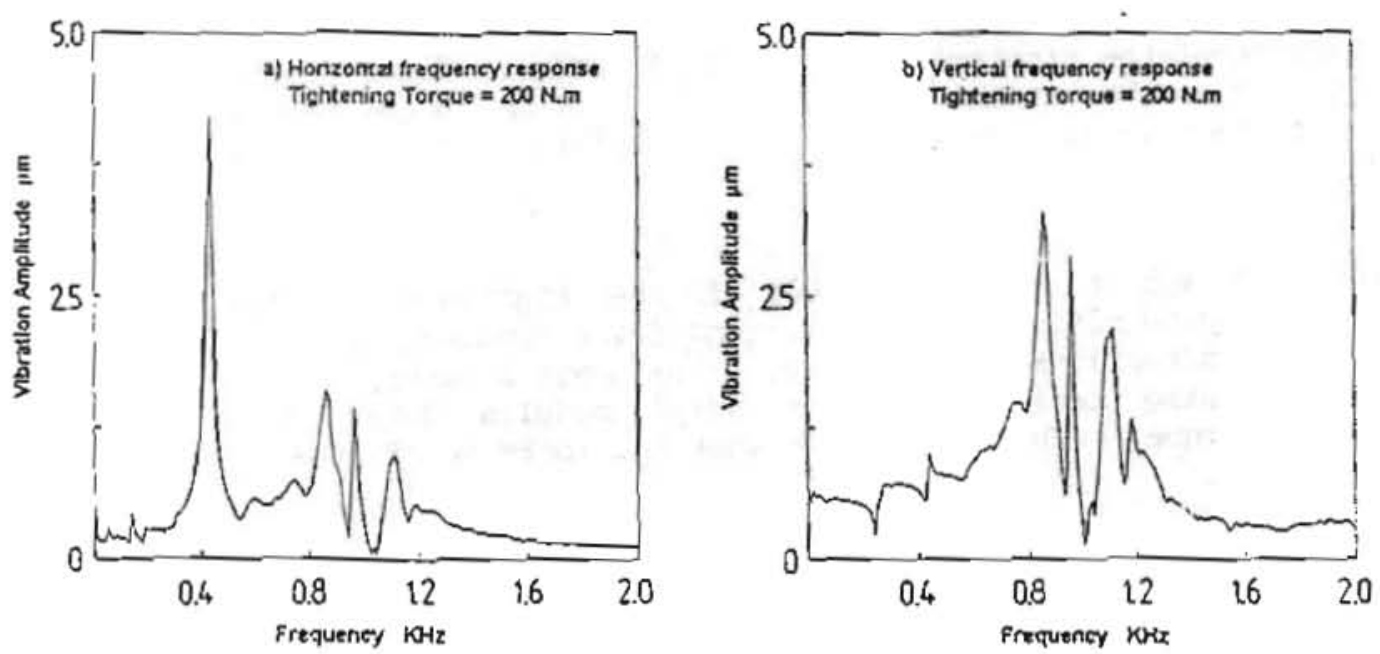

F1g.4 Typical frequency respose of the tooling system with curvic coupling.

Figs, 5 and 6 show the effect of the tightening torque on the natural frequency in the horizontal and vertical directions respectively. For both cases, the natural frequency increases with the tightening torque. The rate of increase in the horizontal direction was found to be about $9 \%$ regardless the coupling module, while in the vertical direction it was about 30\% with the taper joint, and about $45 \%$ for both the flat joint and the curvic coupling. In the horizontal excitation, the tooling system with curvic coupling is superior to those with flat and taper joint modules. However, in the case of vertical excitation, the tooling system with curvic coupling showing higher natural frequencies at higher values of the tightening torques. These results can be such interpreted that the static tightening torque increase significantly the joint module stiffness, particularly of the curvic coupling, and in turn increases the natural frequency. 


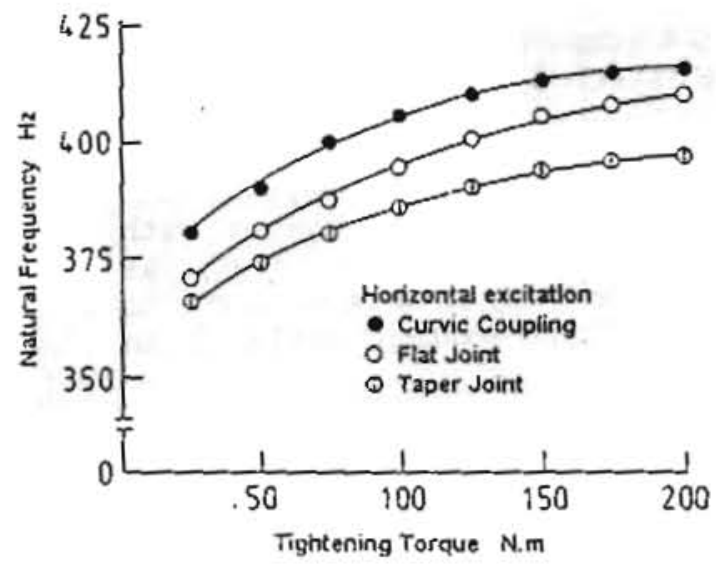

Fig.5 Effect of the tightening torque on the natural frequency in horizontal dir.

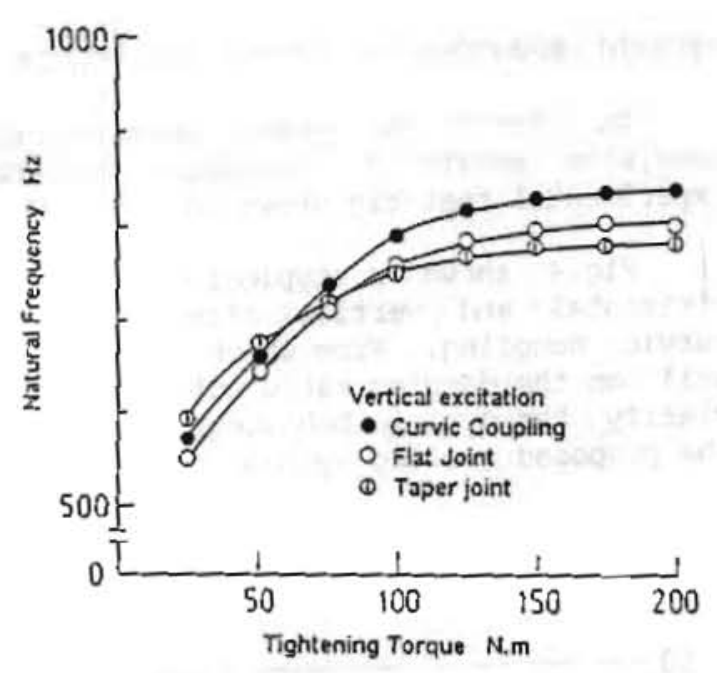

Fig. 6 Effect of the tightening torque on the natural frequency in vertical dir.

Figs. 7 and 8 show the effect of the tightening torque on the calculated damping ratios of the different joint modules, in the horizontal and vertical directions respectively. From these figures, it can be seen that the damping ratio of all the joint modules decreases with the tightening torque. This is because that the increase of the tightening

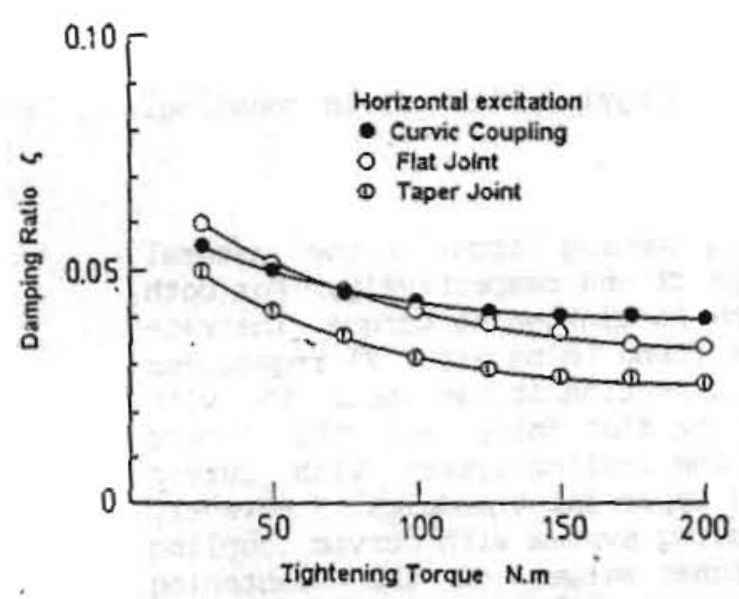

Fig. 7 Effect of the tightening torque on the damping ratio in horizontal dir.

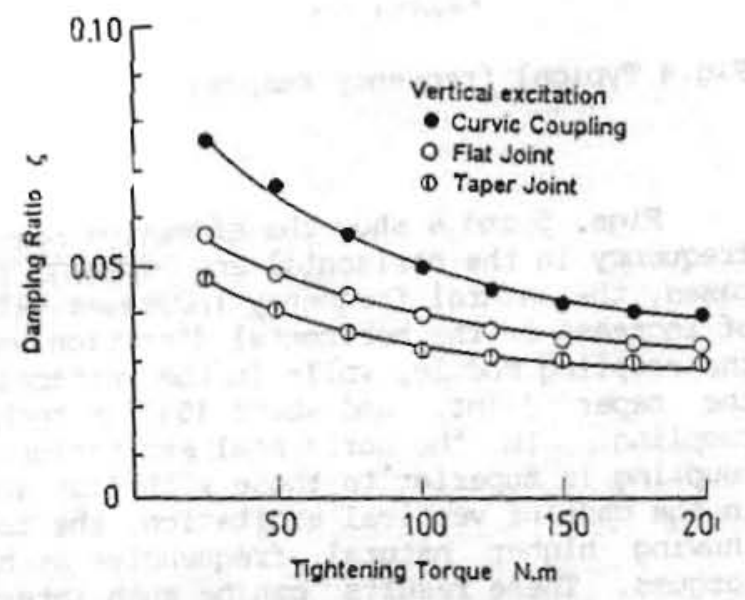

Fig. 8 Effect of the tightening torque on the damping ratio in vertical dir. 
torque may reduce the resulting relative displacement at the joint surfaces, it consequently decreasing the due damping capacity. It is also interesting to point out that the damping capacity, measured as the damping ratio, of the tooling system with curvic coupling is superior to the other joint modules, particularly at high values of the tightening torques.

In addition, Fig. 9 shows the observed improvement rates in the static and dynamic behaviour between the proposed tooling system with curvic coupling and the other joint modules, i.e. the taper joint and the flat joint. From this figure, the tooling system with curvic coupling improves the horizontal bending stiffness by $38 \%$, the vertical bending stiffness by $65 \%$, the horizontal damping ratio by $50 \%$, and the vertical damping ratio by $30 \%$.

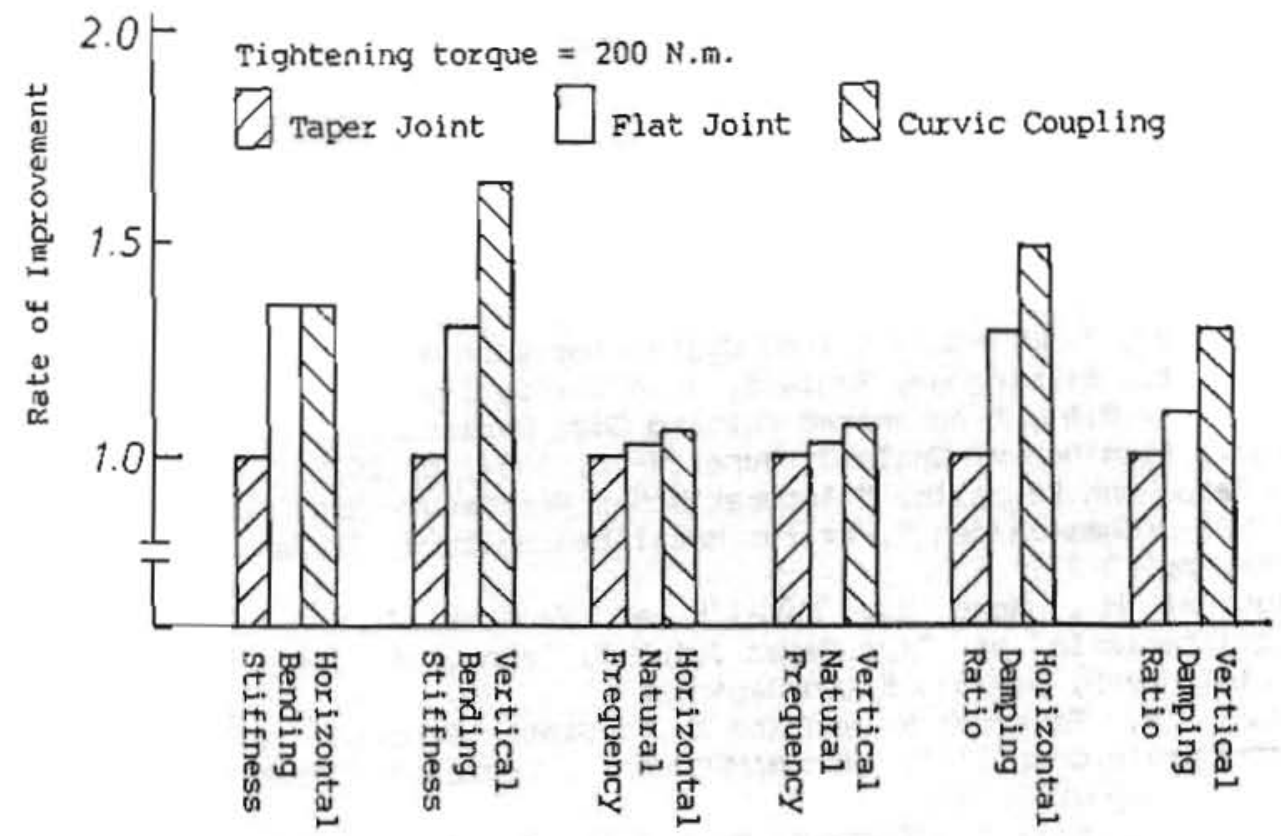

Fig.9 Improvement rates in the static and dynamic behaviour of the proposed tooling system.

To sum up, the static and dynamic characteristic features of the tooling system with curvic coupling are to have higher static bending stiffness, natural frequency and damping capacity than those of the flat and taper joints. When considering the structural configuration of the curvic coupling, these interesting features may be derived from the tooth meshing mechanism of the curvic coupling itself. Such meshing mechanism may allow the considerable micro-slip between the teeth of the two members showing higher damping capacity, simultaneously ensuring high joint stiffness due to the precise meshing mechanism of the coupling teeth. 
CONCLUSION:

In this paper, the static and dynamic behaviour of a proposed tooling system have been experimentally investigated, using as joint modules the curvic coupling, $7 / 24$ taper joint and flat joint, which in the form of vertical-spindle of surface grinding machine.

As a result, it can be clarified that the tooling system with curvic coupling has faverable static and dynamic behaviour. Generally, such system has high possibility of applying it to the automatic tool changing system of such vertical-spindle of the grinding machine, because of its well known accurate locating function and preferable static and dynamic behaviour.

It is, however, noteworthy that the tightening torque of the joint module has a significant effect in determining the static and dynamic behaviour of the tooling system to a great extent.

\section{RFFERENCES:}

[1] Tsutsumi M., Anno Y. and Ebata N., " Static Characteristics of 7/24 Taper Joints for Machining Center ", Bull. of the JSME, Vol.26, No.213, March 1983, pp. 461-467.

[2] Reiter N., " MUTIFLEX- A Tool System for Automatic Turning ", 1st Int. IFS Conf., Birmingham, England, June 26-28, 1984, pp.235-256.

[3] Erickson R.A., " Automated Cutting Edge Replacement ", 1st Int. IFS Conf., Birmingham, England, June 26-28, 1984, pp.261-272.

[4] Wolfgang von Zeppelin, "Automatischer Werkzeugwechsel nach Verschleiß an NC-Drehmaschinen ", tz fur Metallbearbeitung, 78 Jahrg., Heft 3 , 1984, pp. 20-27.

[5] Tsutsumi M., Anno Y., Nakai K. and Yamazaki Y., " Study on the Characteristic of 7/24 Taper Joints", Trans. of the JSME, Vo1.51, No.462, 1985, p. 425-435 (In Japanese).

[6] Hazem S. Tsutsumi M. and Ito Y., " Static Behavior of Turret Head With Curvic Coupling", 13th NAMRC Conf., Berkeley, California, May 1922, 1985, pp. 277-282.

[7] Hazem S., Mori J., Tsutsumi M. and Ito Y., " A New Modular Tooling System of Curvic Coupling Type", 26th Int. MTDR Conf., Manchester, September 17-18, 1986, pp. 261-267.

[8] Hazem S. and Ito Y., " Study on Flexible Spindle-Chuck Modular System of Curvic Coupling Type ", 3rd Int. ASAT Conf., Military Technical College, Cairo, April 4-6, 1989, pp.677-685.

[9] Hazem S. and Ito Y., "Proposal of CACa Methodology For Jointing Method of Curvic Coupling Type ", 4th PEDAC, Alexandria, December 2729, 1989, pp.455-464.

[10] Harris C.M. and Crede C.E., "Shock And Vibration Handbook ", Vol.1, Chpt. 2, McGraw-Hill, 1961. 